\title{
Cultural Competence and Its Associated Factors Among Nurses Working in Tertiary Hospitals: A Cross-Sectional Study
}

\section{Bonsa Amsalu Geleta}

Metu University

Yosief Tsige Radie

Addis Ababa University

Habtamu Abera Areri ( $\sim$ habtamu.abera64@gmail.com )

Addis Ababa University

\section{Research Article}

Keywords: Cultural competence, nurses, tertiary hospital

Posted Date: September 8th, 2021

DOl: https://doi.org/10.21203/rs.3.rs-754525/v1

License: (c) (1) This work is licensed under a Creative Commons Attribution 4.0 International License.

Read Full License 


\section{Abstract \\ Background}

Ethiopian nurses are facing a culturally diverse population predominantly due to the coexistence of multicultural and diverse ethnics in the country. This study aimed to assess the level of cultural competency and its associated factors among nurses working in tertiary hospitals of Addis Ababa, Ethiopia.

\section{Methods}

A cross-sectional study was conducted on nurses working in tertiary hospitals of Addis Ababa city from March 1 to April 30, 2018. A systematic random sampling technique was used to select 352 nurses. Data were collected using an English version self-administered cultural competence questionnaire for nurses scale. Data was entered into Epi data manager version 4.2.2 and exported to SPSS software version 20.0 and analyzed using descriptive statistics and inferential statistics.

\section{Results}

A total of 343 participants were involved in the study with a response rate of $97.4 \%$. The overall cultural competence level of participants was low to moderate $(1.94 \pm 0.65)$ with the highest mean was scored for cultural sensitivity $(2.54 \pm 0.69)$ and the lowest mean was scored for cultural skill $(1.62 \pm 0.98)$. Participants' age, religion, level of education, work experience, current role, spoken languages beside Amharic and English, previous cultural care education, work experience beside the current hospital, the experience of caring for diverse patients and availability of interpreter service were the factors significantly associated with cultural competence at $p<0.05$.

\section{Conclusion}

The overall cultural competence of nurses was low to moderate and influenced by several factors. Therefore, nurses and other responsible bodies should struggle to improve the cultural competence of nurses. Furthermore, a national large-scale study with patient's perspectives was recommended.

\section{Background}

Cultural competence is a set of congruent behaviours, attitudes, and policies that come together in a system, agency, or amongst professionals and enables that system, agency, or those professionals to work effectively in cross-cultural situations [1]. The ultimate goal of cultural competency in nursing care is to reduce health disparity and to provide optimal nursing care to the patient regardless of their race, ethnic background, the native language spoken and religious or cultural belief [2]. Nurses are working in a 
culturally diverse population, mainly because of immigration and globalization in western countries [3]. Medical tourism is another reason for cultural diversity. In Thailand, India, Singapore, and Malaysia, medical tourists are the primary sources of patients' cultural diversity [4]. However, Ethiopian nurses are facing a culturally diverse population predominantly due to the co-existence of multicultural and multiethnic society in the country. Ethiopia is the second-largest African country following Nigeria with one of the most ethnically diverse nations with more than 80 ethnic groups, each with their language, norms, belief, and religion in the world [5]. Besides, Ethiopia is the second-largest refugee-hosting country in Africa, with more than 800,000 refugees from neighboring countries [6].

Studies have indicated that there are considerable shreds of evidence on unequal treatment and the existence of health inequalities or disparities in healthcare among a culturally diverse population. Delivering culturally competent care is a powerful tool to eliminate these disparities in the health status of people of different cultural, racial, and ethnic backgrounds $[7,8]$. As a result of this, globally, cultural competence in nursing care has gained attention and is now recognized by health policymakers, care administrators, academicians, nurses, and nursing researchers as a strategy to eliminate health disparities $[2,9,10]$.

In 2016, the American Nursing Association recognized cultural competence and included it for the first time in the scope and standards of nursing practice [11]. American academy of nursing (AAN) has also developed universally applicable guidelines for implementing culturally competent care, which can serve as a resource for nurses in various roles[12]. However, lack of clarity around the concept of culture, inadequate recognition, and the scarcity of research-based outcomes of cultural competence that provide evidence of efficient strategies are still the limited implementation of cultural competence in nursing practice [2]. Besides, the study shows that many nurses have given minimal or no attention to monitoring the cultural competency of their own and members, even if the standard of culturally congruent nursing care. In nursing practice and other guidelines for implementing culturally competent care persuaded them to be culturally relevant [13].

Therefore, assessing the level of cultural competence among nurses and taking appropriate action is one strategy to increase the cultural competency of nurses in nursing practice. Many studies exist that examine the cultural competency of nurses. However, the majority of these studies have been conducted in the United States, Canada, UK, and Australia, while only a few studies were conducted in Africa. The findings of these studies were inconsistent in which cultural competence of nurses was low in South Africa, Israel, Taiwan, and Thailand [4, 14-16], moderate in Italia and Iran $[17,18]$ and high in the USA and Saudi Arabia $[19,20]$. These studies also showed that different factors could influence the cultural competence of nurses, such as level of education, nurses' year of experience, one's ethnicity age, sex, and frequency of caring for culturally diverse clients $[4,15,16,20]$.

Despite the existence of a diverse socio-cultural society that needs nurses to be culturally competent, cultural competence among nurses is not studied. Therefore, this study investigated the level of cultural competence and its influencing factors among nurses working in tertiary hospitals. 


\section{Theoretical framework}

Papadopoulos, Tilki, and Taylor (PTT) model guided this study. PTT model is one of the nursing theories that facilitate understanding of current nursing care practices and patients' demand for nursing care regardless of their cultural, religious, and linguistic background. It describes the nurses' capacity to provide effective nursing care that takes the patient's cultural beliefs, behaviours into consideration. According to this model, to be culturally competent, every nurse must pass through four stages of the PTT model. These stages are cultural awareness (first stage), cultural knowledge (second stage), cultural sensitivity (third stage), and cultural skill (fourth stage[21]. These four sub-constructs of cultural competence have been commonly recognized and used in different studies $[16,22,23]$. Therefore, the present study also used the four sub-constructs of the PTT model for developing cultural competence.

\section{Methods}

\section{Study area, period and design}

A cross-sectional study study was conducted at the three largest tertiary hospitals in the capital city and home to almost all ethnic groups and cultural centres. It also harbours a large population of foreign residents like Eritrean, Kenyan, Sudanese, and Djiboutian [5]. The study period spanned from March to April 2018.

\section{Sample Size Determination And Sampling Procedure}

The sample size was calculated using a single population proportion formula [24] by considering $50 \%$ as a proportion of nurses' cultural competence to maximize sample size since no studies are showing the cultural competencies of the nurses in the country. Therefore, the initial sample size was 384 . However, the total number of nurses was $2069(<10,000)$, then we could use sample correction, and the sample size estimated was 320 . Then, a $10 \%$ non-response rate was considered, and the final sample size was 352. Then after, selecting study participants, a systematic random sampling technique was used. First, the total sample size was allocated to hospitals proportional to the number of nurses in each hospital. Then, an individual nurse in each hospital was selected by systematic random sampling technique every 6th interval by identifying an initial starting nurse by using a lottery method.

\section{Data collection tool}

For this study, the English version, structured, and self-administered cultural competence questionnaire for nurses (CCQN) was used. The survey tool was designed by principal investigators to measure the cultural competence of nurses working in hospitals based on the Papadopoulos, Tilki, and Taylor (PTT) model that consists of four constructs of cultural competence[21]. These included cultural awareness, cultural knowledge, cultural sensitivity, and cultural skill. The questionnaire was content validated by five 
nurse experts from different universities in the country. A pre-test was conducted on 36 nurses two weeks before actual data collection to test the internal consistency of the tool and see the ambiguity of the word and clarity of the sentence of the items. Internal consistency was tested using a reliability scale. The results showed strong reliability for all subscales and overall scale with Cronbach's alpha value of 0.92 , $0.85,0.96,0.90$ for cultural awareness, cultural knowledge, cultural sensitivity, and cultural skill subscales respectively and 0.94 for the overall scale. Based on participants' comments, unclear and jargon words were modified. The internal consistency of the four subscales and the overall CCQN were also tested on an actual study using a reliability scale. The finding indicated that overall, CCQN had strong reliability with Cronbach's alpha of 0.92 , and the individual subscales ranged from 0.75 for cultural sensitivity to 0.92 for cultural skill.

\section{Measurement}

The level of each subscale (cultural awareness, cultural knowledge, cultural sensitivity, and cultural skill) and overall cultural competence was categorized based on previous studies into low level, low to a moderate level, moderate level, and high level $[5,16,23,25]$. If a mean score of each subscale and overall cultural competence is $0-1,>1-2,>2-3$, and $>3$, the level of competency was categorized as low level, low to a moderate level, moderate level, and high level respectively.

\section{Data analysis}

The coded data was entered into Epi-data version 4.2.2 and exported to statistical package for the social science (SPSS) Version 20.0. The data was then analyzed using descriptive statistics, independent t-test, and one-way ANOVA. Before using these statistical tests, data were tested for the assumption. The result indicated that there were no non-normally distributed variables except cultural competence scores across groups of work experience and the current role of participants. Because of this, the Kruskal-Wallis test was used to examine the mean score differences between groups of these two variables. The assumption of homogeneity of variance was examined by Levene's test of equality of variance [24]. The results indicated that there were no significantly different variances among groups. The statistical significance was set at $p<0.05$.

\section{Results}

\section{Participant's characteristics}

Out of the total participants involved, 343 participants returned the questionnaire making a response rate of $97.4 \%$. More than half $53.1 \%(n=182)$ participants were males. The mean age of the participants was 28.51(SD, 4.22). Amhara $45.2 \%(n=155)$ by ethnicity and Orthodox $60.9 \%(n=209)$ by religion dominates study participants. The majority $89.2 \%(n=306)$ of the study participants were Bachelor's degree holders in nursing. Almost three-quarters of $72.9 \%(n=250)$ of participants reported that they had no experience in learning concepts of cultural care nursing in their previous nursing education.

Conversely, more than half, $54.5 \%(n=187)$ of them had experience in caring for patients from culturally, 
and ethnically diverse groups with $62.9 \%(n=117)$ of them had an opportunity to care for these patients almost every day (Table 1)

\section{Cultural competence of participants}

The overall cultural competence of participants was $1.94(S D=0.65)$ out of a total possible score range from 0 to 4, which indicates a low to moderate level of cultural competency. Similarly, the level of cultural competence of participants for cultural knowledge $(M=1.91, S D=0.76)$ and cultural skills $(M=1.62, S D$ $=0.98)$ subscales was low to moderate. The lowest mean score was observed for the cultural skill scale $(M=1.62, S D=0.98)$ and the highest score was observed for the cultural sensitivity scale $(M=2.54, S D=$ $0.69)$ (Table 2).

The finding of this study also revealed that almost half, $49.9 \%(n=171)$ (of participants were in the low to moderately competent on the overall CCQN scale score. The highest percentages of participants were in low to moderate for cultural awareness and knowledge, with $42.6 \%(n=146)$ and $38.5 \%(n=132)$, respectively (Fig. 1).

\section{Factors associated with cultural competence of participants}

There was a statistically significant difference among participants by language spoken beside Amharic and English languages $(t=3.53, p<.001)$, previous cultural care education in nursing programs $(t=8.13$, $p<.001)$, the experience of caring for culturally and ethnically different patients $(t=8.40, p<.001)$, work experience in other health institution other than their current hospital $(t=8.30, \mathrm{p}<.001)$ and interpreter service $(t=8.28, p<.001)$. The computed Cohen's $d$ using the value of $t$-test statistics revealed a large effect based on Cohen's standard for all these variables except for languages spoken beside Amharic, and English was a small effect. However, no significant difference was noted between mean score of males $(M=1.93, S D=0.67)$ and female $(M=1.94, S D=0.64) ; t=-.05, p=.65, d=-.004$. The finding signifies that being male or female does not have an impact on the cultural competence level of participants (Table 3 ).

One-way AVOVA revealed that there was no significant difference in mean scores among groups by ethnicity $[F(3,342)=0.77, p=.51]$, language background $[F(4,161)=1.46, p=.21]$, frequency of caring for patients culturally and ethically different from one's own $[F(3,183)=2.26, p=.08]$, level of health institution served $[F(3,117)=0.13, p=.94]$, and type of interpreter used $[F(2,137)=0.06, p=.97]$. However, there was a statistically significant difference in mean scores among groups by age $[\mathrm{F}(2,340)$ $=7.81, p<.001, \eta 2=.04]$, religion $[F(2,340)=3.04, p=.029]$, education level $[F(2,340)=6.10, p=.002]$. Further, post-hoc comparisons using the Tukey's honestly significant difference (HSD) test was conducted for these variables (Table 4).

The Kruskal-Wallis test indicated that there was statistical significance difference in mean score across the work experience groups $\left(X^{2}(3)=24.64, p<0.001, \eta^{2} H^{2}=0.06\right)$ and across the groups by current role of 
participants $\left(X^{2}(3)=15.4, p=0.001, \eta^{2}{ }_{H}=0.03\right)$. Calculated eta squared using Kruskal-Wallis test statistics indicated that the variance in mean score among groups by work experience was medium $\left(\eta^{2} H\right.$ $=0.06)$, and by current role was small $\left(\eta^{2}{ }_{H}=0.03\right)$ (Table 5).

\section{Discussion}

The finding of the present study indicated that the overall cultural competence of participants was low to moderate. These findings were consistent with many recent studies conducted in Taiwan, Thailand, South Africa and Israel in which a small percentage of nurses were involved in culturally appropriate nursing care $[4,14-16]$. In contrast to the present findings, the cultural competence level of the nurse in Italia and Iran was moderate $[17,18]$. The obstetric and neonatal nurses practising in the United States was moderately high [19], and expatriate nurses in Saudi Arabia was high [20] in which they had better cultural competence than the present finding. The differences could be due to limited experience and training cultural competencies among participants. Providing adequate cultural competence education for nursing students and designing guidelines and standards for cultural competence that could help and guide nurses in providing culturally congruent nursing care.

Knowledge and skills subscales are an essential stage of cultural competence in becoming culturally competent[26]. However, the present finding revealed that participants scored lower mean on cultural knowledge and skills and slightly higher on cultural awareness and sensitivity. This finding is similar to the study conducted in Taiwan and Japan $[16,27]$. It reflects that participants' cultural knowledge and skills level were not encouraged to become knowledgeable about their patient's cultural beliefs, values, and practices, and skilful in cultural and linguistic appropriate assessment to collect relevant data from a diverse patient.

The present study found a significant difference in the mean score of cultural competence based on participants' age groups in which older nurses scored higher than younger nurses. Studies in Thailand and Canada reported similar findings that cultural competence was positively associated with age $[4,28]$. The reason for this may be older nurses had more opportunities for exposure to different cultures than younger nurses. Participants with master's degree nurses scored significantly higher than those with a diploma and bachelor's degree in nursing. This finding is consistent with another published study in Carolina[29]. It indicates that additional continuing education leads to a significant difference in the level of cultural competencies of nurses. The reason could be due to trans-cultural nursing education in a postgraduate curriculum that may benefit masters degree nurses to have higher cultural competence.

This study also revealed the relationship between years of nursing work experience in their current hospital and the cultural competence of nurses as measured by the CCQN score. Studies in Israel, Italia, Iran, and Saudi Arabia reported a similar finding that the mean scores of nurses increased with years of experience $[15,17,18,20]$. The participants who speak only Amharic and English language scored lower than those who can speak other languages. Therefore, providing culturally competent nursing care is easier for nurses who speak languages besides Amharic and English than those who cannot speak. The 
finding is congruent with a study conducted in South Africa and Korea that language proficiency was associated with the cultural competence of nurses $[4,14,15,30]$. Thus, the researcher suggested that it is better if hospitals design strategies and policies that enhance recruitment and retention of the diverse nursing workforce that mirror images with patients' populations based on their language proficiency.

The previous study found that cultural competence was associated with the position of a nurse [30]. The present study also supports a finding that a statistically significant difference in mean cultural competence scores between groups of participants by the current role. Prior studies found that having experience of caring for diverse clients have a considerable impact on nurses' cultural competency [27]. The finding is consistent with the present study that the experience of caring for patients culturally and ethnically different from one's own improves the cultural competence of nurses. The possible explanation for this may be their exposure to patients from diverse cultural backgrounds might positively influence their cultural competence. Nursing theorist suggested that exposure to culturally and ethnically diverse populations play an important role in improving cultural competency levels[26, 31].

The present finding did not find a significant difference in a mean score of cultural competencies based on the frequency of caring for patients culturally and ethnically different from one's group. Incongruent to this, the prior study showed that nurses who had cared for clients with different cultural backgrounds on a more frequent basis had the highest mean scores of cultural competencies on NCCS when compared with a nurse who had less frequent caring experiences [16]. A possible explanation for this may be due to different measurement scales of cultural competence of nurses.

Participants who had work experience in another institution were more culturally competent than their counterparts. The reason could be due to their previous exposure to culturally diverse groups positively influenced their cultural competence. However, no study examined the association between the work experience of nurses other than in their current hospital and cultural competence. Thus, further research investigating the relationship between this variable is needed to confirm the present result.

The present study found that participants who used an interpreter for the language they are not fluent were scored high than those who did not use it. The finding supports the literature that indicates using interpreters improve the cultural competence of nurses[11, 12]. It is recommended that interpreters must ensure confidentiality, be knowledgeable about the health care language, and ethically conduct all sessions[32]. In addition to this, communication with patients in their language with qualified interpreters improves patients' understanding of their diseases and treatment processes as well as generating trust in providers [33]. However, in the present study, only $42 \%$ of participants were used interpreters for the languages they were not fluent in which none of them used trained interpreters. The researchers concluded that the limited availability of qualified interpreter services compounded in difficulties of providing culturally congruent nursing care. The family members and others else should be used only as a last option when trained interpreters and translators are not available due to potential risks of privacy issues and bias in interpretation. Thus, the provision of regularly available trained interpreters/translators 
as well as language training for nurses is, therefore, an essential component in improving the cultural competence of nurses.

In contrast to other several studies throughout the globe, the finding of this study showed that few participants had ever learned cultural care nursing and no participants who reported prior cultural care nursing training $[17,18,34]$. The possible explanation for this could be inadequately integrated cultural nursing care in the nursing education curriculum, and attention has not been given to cultural competence in the country.

\section{Conclusion}

The overall cultural competence of nurses was at a low to moderate level, which may result in less likely to meet the needs of culturally, ethnically, and linguistically diverse patients. Age, religion, education level, languages spoken besides Amharic and English, work experience in the current hospital, work experience besides existing hospital, current role, knowledge of caring for patients culturally and ethnically different from one's own, availability of interpreter service, and previous cultural related education were the identified factors that influence cultural competence of nurses. Therefore, nurses and other responsible bodies should struggle to improve the cultural fitness of nurses to provide culturally congruent nursing cares that is necessary for meeting the needs of diverse patients in the country. Lastly, a national representative study with patient's perspectives, including the cultural competence of nurses working in a more geographically diverse area. The more heterogeneous health care providers that would bring more significant implications for policymakers and health care practitioners was recommended.

\section{Relevance To Clinical Practice}

Cultural competency is a critical aspect of nursing practice. Nurses working in a diverse society should learn and apply the concept of culturally congruent care. There is clear evidence of unequal treatment in various cultural settings. The curriculum for nursing education given less emphasis to congruent cultural care, and this was affecting the clinical nursing practice concerning respecting, accepting and implementing culturally congruent clinical practices. Therefore, nurses working in diverse cultural settings should be culturally competent.

\section{List Of Abbreviations}

\section{AAN}

American Academy of Nursing, ANOVA:Analysis of Variance, CCQN:Cultural Competence Questionnaire for Nurses, PTT:Papadopoulos, Tilki and Taylor, UK:United Kingdom, USA:United States of America

\section{Declarations}

\section{Ethics approval and consent to participate}


To conform the Declaration of Helsinki (1964) and Population Screening Act, ethical approval was obtained from the institutional review board [AAU/chs/ahnsg03-2018]. Permission was obtained from the hospital management. Written informed consent was obtained from participants after they got informed about the objectives of the study, and their right to refuse and respond fully or partially to the questionnaire was respected.

\section{Consent for publication}

Not applicable.

\section{Availability of data and materials}

The datasets used and/or analysed during the current study are available from the corresponding author on reasonable request.

\section{Competing interests}

The authors declare that they have no competing interests.

\section{Funding}

Addis Ababa University granted data collection fund. The university had no role in designing the study, data collection, analysis, and interpretation of the study.

\section{Authors' contributions}

BAG designed the study, collected data, analysed and interpreted data, and wrote and revised the manuscript. YTR advised in developing the study, interpreted the data, and critically revised the manuscript. HAA advised in designing the study, interpreted the data, and critically revised the manuscript. All authors read and approved the final version of the paper.

\section{Acknowledgments}

The authors acknowledge Addis Ababa University for supporting the study. The acknowledgement also extends to the Research Ethics Committee of the University and management of selected referral hospitals. Finally, we would also like to thank the study participants.

\section{References}

1. Leininger M, M., McFarland M, R.: Transcultural nursing: Concepts, theories, research and practice, 3rd edn. New York: McGraw-Hill; 2002.

2. Thackrah R, Thompson S: Refining the concept of cultural competence: building on decades of progress.. Medical Journal of Australia 2013, 199(1):35-38.

3. The Process of Cultural Competence in the delivery of Healthcare Services transcultural care net 
4. Bunjitpimol P, Somrongthong R, Kumar R: Factors affecting nursing cultural competency in private hospitals in Bangkok, Thailand. International Journal of Healthcare 2016, 2(1).

5. CSA. E: Population projection of Ethiopia for all Regions at wereda level from 2014-2017. In.; 2014.

6. Refugee report

7. Kaur B: Cultural Competent Care in Hong Kong.. International Journal of Social Science and Humanity 2016, 6(2):136.

8. Baghdadi $\mathrm{N}$ : Cultural competency of nursing faculty teaching in baccalaureate nursing programs in the US. In. Boston, Massachusetts, USA Northeastern University; 2015.

9. Betancourt J, R., Green A, R., Carrillo J, E., Owusu Ananeh-F I: Defining cultural competence: a practical framework for addressing racial/ethnic disparities in health and health care.. Public health reports 2016.

10. Betancourt J, R., Green A, R., Carrillo J, E., Park E, R.: Cultural competence and health care disparities: key perspectives and trends.. Health affairs 2005, 24(2):499-505.

11. Marion L, Douglas M, Lavin M, Barr N, Gazaway S, Thomas L: Implementing the new ANA standard 8: Culturally congruent practice.. The Online Journal of Issues in Nursing 2016, 22(1).

12. Douglas MK, Rosenkoetter M, Pacquiao D, F., Callister L, Hattar-Pollara M, Lauderdale J: Guidelines for implementing culturally competent nursing care. Journal of Transcultural Nursing 2014, 25(2):109-121.

13. Saha S, Korthuis P, Cohn J, Sharp V, Moore R, Beach M: Primary care provider cultural competence and racial disparities in HIV care an outcomes.. Journal of General Internal Medicine 2013, 38:622629.

14. DE Beer J, Chipps J: A survey of cultural competence of critical care nurses in KwaZulu-Natal.. Southern African Journal of Critical Care 2014, 30(2):50-54.

15. Orit E: Cultural Competence Among Hospital Nurses in Israel In. Romania University of Babes-Bolyai Cluj; 2013.

16. Lin C, N., Mastel-Smith B, D. A, Y-H. L: Cultural competence and related factors among Taiwanese nurses. Journal of Nursing Research 2015, 23(4):252-261.

17. Cicolini G, Della PC, Comparcini D, Tomietto M, Cerratti F, Schim S, M.: Cultural competence among Italian nurses: A multicentric survey.. Journal of Nursing Scholarship 2015, 47(6):536-543.

18. Bastami M, R., Kianian T, Borji M, Amirkhani M, Saber S: Assessment of cultural competence among nurses.. Medical Ethics Journal 2016, 10(36):65-72.

19. Heitzler E, T.: Cultural Competence of Obstetric and Neonatal Nurses.. Journal of Obstetric, Gynecologic \& Neonatal Nursing 2017, 46(3):423-433.

20. Inocian E, P.: A Baseline Assessment Survey on Cultural Competency among Expatriate Nurses in Saudi Arabia.. International Journal of Nursing 2015, 4(1).

21. Papadopoulos I, Tilki M, Lees S: Promoting cultural competence in health care through a research based intervention in the UK.. Diversity in Health and Social Care 2004, 1(2):107-115. 
22. D.H., C-YL. C: Development and Psychometric Evaluation of the Korean Version of the Cultural Competence Scale for Clinical Nurses.. Asian Nursing Research 2014, 8 305-312.

23. Marzilli C: Assessment of cultural competence in Texas nursing faculty.. Nurse education today 2016, 45:225-229.

24. Daniel WW: Biostatistics: a foundation for analysis in the health sciences, 8th edn. New York: Wiley; 2010.

25. Songwathana P, Siriphan S: Thai Nurses' Cultural Competency in Caring for Clients Living in a Multicultural Setting.. Pacific Rim International Journal of Nursing Research 2015, 19(1):19-31.

26. Campinha-Bacote $\mathrm{J}$ : The process of cultural competence in the delivery of healthcare services: $\mathrm{A}$ model of care.. Journal of transcultural nursing 2002, 13(3):181-184.

27. Kawashima A: Study on cultural competency of Japanese Nurses. An unpublished dissertation.. In. Fairfax, Virginia: George Mason University; 2008.

28. Almutairi AF, Adlan AA, Nasim M: Perceptions of the critical cultural competence of registered nurses in Canada.. BMC nursing 2017, 16(1):47.

29. Lampley T, Little K, Xu Y: Cultural competence of North Carolina nurses. a journey from novice to expert Home Health Care Management \& Practice 2008, 20(6): 454-461.

30. Chae D, H., Park Y, H., Kang K-H, Lee T: A Study on Factors Affecting Cultural Competency of General Hospital Nurses.. J Korean Acad Nurs Admin 2012, 18(1):76-86.

31. Leininger MM: What is transcultural nursing and culturally competent care.. Journal of transcultural nursing 1999, 10(9).

32. Giger J, Davidhizar R, Purnell L, Harden T, Phillips J, Strickland O: American Academy of Nursing expert panel report: Developing cultural competencies to eliminate health disparities in ethnic minorities and other vulnerable populations.. Journal of Transcultural Nursing 2007, 18(2):100.

33. Jacobs E, Lauderdale D, Meltzer D, Shorey J, Levinson W, Thisted R: Impact of interpreter services on delivery of health care to limited-English-proficient patients.. Journal of General Internal Medicine, 2001, 16(7):468-474.

34. Sagar PL: Transcultural nursing theory and models: Application in nursing education, practice, and administration: Springer Publishing Company; 2011.

\section{Tables}

Tables 1-5 are not available with this version.

\section{Figures}




\section{Image not available with this version}

Figure 1

Figure 1 